Chirurgia (2017) 112: 457-468

No. $4, \quad$ July - August

Copyright $\odot$ Celsius

http://dx.doi.org/10.21614/chirurgia.112.4.457

\title{
Results of 1-year Diet and Exercise Interventions for ER+/PR \pm /HER2- Breast Cancer Patients Correlated with Treatment Type
}

\author{
Diana V. Artene ${ }^{1}$, Cristian I. Bordea², Alexandru Blidaru ${ }^{3}$ \\ "'Carol Davila" University of Medicine and Pharmacy, Bucharest, Romania \\ 2Department II of Surgical Oncology, "Prof. Dr. Al. Trestioreanu" Institute of Oncology, Bucharest, Romania \\ ${ }^{3}$ Surgical Oncology Department, "Prof. Dr. Al. Trestioreanu" Institute of Oncology, Bucharest, Romania \\ "Carol Davila" University of Medicine and Pharmacy, Department of Surgery, Oncological Surgery Chair
}

Corresponding author:

Diana Artene, ND, MNS, PhD

Gheorghe Tițeica street, no.178

Bucharest, Postal Code 020304

Romania

E-mail: diana.artene@gmail.com

\section{Rezumat}

Rezultatele unei interventii de dietă + exercitii cu durata de 1 an în cazul pacientelor cu cancer mamar ER+ corelate cu tipul de tratament

Scop: Multe paciente cu cancer mamar prezintă creştere ponderală pe parcursul administrării chimioterapiei sau al tratamentului antiestrogenic, ceea ce creşte riscurile de limfedem, metastază, recurență şi mortalitate de cauză general şi specific oncologică. Studiul de față îşi propune să evalueze eficiența unei intervenții nutriționale şi kinetice în contracarearea obezității pacientelor cu cancer mamar.

Pacienți şi metodă: 165 paciente cu cancer mamar ER+/PR \pm / HER2aflate în tratament antiestrogenic au fost randomizate pentru a urma timp de 1 an, la domiciliu, fie o dietă bazată pe alimente natural bogate în proteine, calciu, probiotice şi prebiotice (D), fie dieta şi 4 minute de exerciții fizice izometrice (D+Ex). Am măsurat greutatea (G), adipozitatea subcutanată (AS) şi adipozitatea viscerală $(\mathrm{AV})$ cu un cântar cu impedanță bioelectrică cu multifrecvențe la 6 şi 12 luni şi am corelat rezultatele cu tipul de chimioterapie, intervenție chirurgicală şi tratament antiestrogenic. Rezultatele au fost analizate folosind testul Friedman, respectiv testul Wilcoxon signed-rank în cazul în care testul Friedman a fost semnificativ statistic.

Rezultate: Ca şi grup, atât pacientele din lotul D+Ex, cât şi pacientele din lotul D au obținut o scădere semnificativ statistic a greutății şi adipozității. Pacientele din lotul D au obținut o scădere a greutății
Received: 03.04.2017

Accepted: 10.05.2017 
de $3,3 \mathrm{~kg}$, a adipozității subcutanate de $3,2 \%$ şi a adipozității viscerale de $1 \%$. Pacientele din lotul D+Ex au obținut o scădere a greutății de $6,5 \mathrm{~kg}$, a adipozității subcutanate de $3,3 \%$ şi a adipozității viscerale de $2 \%$. În lotul D+Ex nu au apărut diferențe semnificative statistic în funcție de tipul de chimioterapie, intervenție chirurgicală sau tratament antiestrogenic. Pacientele din lotul D cu mastectomie şi inhibitori de aromatază au obținut rezultate semnificative statistic atât pentru scăderea greutății cât şi pentru scăderea adipozității subcutanate şi viscerale. Dar pacientele din lotul D cu tratament chirurgical mamar conservator, chimioterapie administrată postoperator sau atât pre cât şi postoperator şi cele în tratament cu Tamoxifen au obținut rezultate semnificative statistic doar pentru pierderea în greutate. Pacientele din lotul D cu chimioterapie neoadjuvantă au obținut rezultate semnificative statistic şi pentru scăderea adipozității viscerale.

Concluzie: Dieta propusă este eficientă pentru pacientele cu cancer mamar ER+/PR $\pm /$ HER2- în tratament antiestrogenic. Adăugarea unui program minim de exerciții fizice este esențială pentru a îmbunătății şansele pacientelor de a contracara obezitatea sarcopenică.

Cuvinte cheie: cancer mamar, obezitate sarcopenică, nutriție oncologică, exerciții izometrice

\section{Abstract}

Purpose: Many breast cancer patients gain weight during chemotherapy and antiestrogenic treatment increasing recurrence, oncologic specific and all-cause mortality risks.

Patients and Methods: $165 \mathrm{ER}+/ \mathrm{PR} \pm /$ HER2- breast cancer patients under antiestrogenic treatment were randomly assigned to follow an at-home diet based on food naturally high in proteins, calcium, probiotics and prebiotics (D), or this diet and 4' isometric exercises (D+Ex) for 1 year. We measured weight (W), body (BF) and visceral fat (VF) using a multi-frequency bioelectrical impedance scale on the $6^{\text {th }}$ and $12^{\text {th }}$ month and we correlated results with chemotherapy, surgery and antiestrogenic medication type. Results were analysed using the Friedman Test, then with Wilcoxon signed-rank tests if Friedman Test was significant.

Results: Overall, the patients' 1-year results show that both D+Ex and D patients obtained statistically significant weight loss and fat loss. D patients lost $3.3 \mathrm{~kg}, 3.2 \% \mathrm{BF}$ and $1 \%$ visceral fat. D+Ex patients lost $6.5 \mathrm{~kg}, 3.3 \% \mathrm{BF}$ and $2 \%$ visceral fat. D+Ex patients obtained statistically significance for $\mathrm{W}, \mathrm{BF}$ and $\mathrm{VF}$ regardless of chemotherapy, surgery or antiestrogenic treatment type. D patients with mastectomy or with aromatase inhibitors lost W, BF and VF. D patients with conservatory surgery, adjuvant or both neoadjuvant and adjuvant chemotherapy and those on Tamoxifen only lost W. D patients with neoadjuvant chemotherapy also lost VF.

Conclusion: This diet is effective for $\mathrm{ER}+/ \mathrm{PR} \pm / \mathrm{HER} 2$ - breast cancer patients on antiestrogenic medication. Adding at least a minimal exercise protocol improves patients' chances of counteracting sarcopenic obesity.

Key words: breast cancer, sarcopenic obesity, oncology nutrition, isometric exercise

\section{Introduction}

Breast cancer patients, who gain weight during treatment, have a higher risk of all cause and oncologic specific mortality, de novo carcinogenesis and recurrence. (1)
Besides, overeating or eating regardless of physical hunger, which are the main weight gain causes - insulin and leptin resistance, dysbiosis and dyslipidemia - are potentially aggravated during breast cancer chemotherapy by sarcopenia. (2) In addition, studies prove 
that sarcopenic obesity which develops during chemotherapy, is present even 3 years after chemotherapy treatment. $(3,4)$

Sarcopenia is not the main cause of weight gain during breast cancer chemotherapy, but it is aggravated by the following, major weight gain causes:

- Induced menopause - which accentuates muscle protein catabolism, generating the decrease in active motor units and type II muscle fibres atrophy, (5)

- Insulin resistance - generated by sedentariness or by overeating and causing triglycerides accumulation in miocytes as well as decreased sarcolemma GLUT4 expression, (6)

- Dyslipidemia - either caused by the eating behaviour or by chemotherapy per se generates leptin resistance manifested by a decreased ability to perceive satiety, (7)

- Intestinal Dysbiosis - directly generated as a chemotherapy side effect - cause modified intestinal permeability (increased for improperly digested proteins and decreased for disaccharides) which induces bloating, cramps, constipation or diarrhea, that may disturb the eating behaviour and eventually lead to insulin resistance and weight gain. (8)

These main weight gain causes are worsened by sarcopenia through basal metabolic rate decrease, and sustained by the eating behaviour and sedentariness of the patient, generating:

- An increased body fat percentage and insulin resistance in patients who do not overeat, (9)

$$
\mathrm{Or}
$$

- An increased body fat percentage and increased insulin and leptin resistance, dysbiosis and dyslipidemia in patients with excessive carbohydrate consumption. (10)

Compensating these etiological weight gain factors, can be hard during chemotherapy, because many patients feel ill, anddue to the fact that, besides studies proving exercise safety during treatment, many oncologists do not recommend physical exercise, neither during nor after the breast cancer treatment. (11)
Nutritionally, both sarcopenia and overeating can be decreased by a high protein diet, through improvement of the muscle protein synthesis/ degradation ratio, through an improved insulin sensitivity and satiety, as well as by influencing the postprandial secretion of insulin, (2) ghrelin, (12) and the main satiety hormones: GLP-1, cholecystokinin and peptide Y. (13)

Dyslipidemia and leptin resistance can be improved by a daily intake of foods, high in omega-3 fatty acids such as fish, cold pressed extra virgin olive oil, rapeseed oil or canola oil, avocado, and various raw seeds, almonds and nuts, and by avoiding soft drinks (high in colorants and high fructose corn syrup), fried food and any food with hydrogenated fats on the ingredients list.

Dysbiosis can also be ameliorated by a daily intake of foods high in prebiotics such as whole grain cereals, beans, lentils, fresh fruits and vegetables, and probiotics such as yoghurt, kephir and sour milk.

Probiotics also have antiproliferative effects on tissues with non-intestinal localization, like breast and prostate, improving estrogenic metabolism and inactivating carcinogenic substances. (14) Comparative studies between probiotic supplements and the one of fermented dairy foods prove that dairies have higher anticarcinogenic effects, because they also contain beneficial metabolites produced by probiotics during milk fermentation. (15)

Fermented dairies also ensure a highly bioavailable calcium intake, important for the protective impact of calcium and vitamin D sensing receptors, key regulators of cell proliferation, differentiation and apoptosis. Studies prove, that a high calcium intake may associate with better breast cancer prognostics. (16) Also, a meta-analysis of 11 studies demonstrates that a high calcium intake decreases breast cancer risk by $19 \%$, as decreasing this risk is extremely important in preventing recurrence or de novo carcinogenesis in breast cancer survivors. (17)

Kinetically, the regular practice of physical exercise during breast cancer treatment sustains a better prognostic (18). Resistance exercises are the most effective ones for treating both sarcopenia (19) and the other weight gain 
causes (20), but are difficult to do after breast cancer surgery without a physical therapist supervision.

Whole body balance isometric exercises are not as effective as resistance exercises because they cannot counteract insulin resistance, leptin resistance, dyslipidemia or dysbiosis (21). Nevertheless, they can prevent skeletal muscle loss (22), they can be practiced even during days when the patient feels ill. Moreover, after learning the proper way to execute them, they can be safely done without monitoring, and they take very little time which is a major argument to convince most patients to easily accept to do them.

Isometric exercises have an anaerobic-like effect improving muscle protein turn-overtowards maintaining active skeletal muscle mass (23). Finally, if we add these muscle protective effects to a high protein diet which is meant to counteract insulin and leptin resistance, dyslipdemia and dysbiosis we can achieve fat loss without muscle loss despite antiestrogenic medication administration.

As for the safety of breast cancer patients practicing physical exercises, one meta-analysis of 51 studies performed in the last 25 years proves that low and moderate intensity physical exercise is safe and beneficial (24).

\section{Method}

\section{Purpose}

This home-based study aims to answer three questions:
1. Is a high protein diet effective for fat loss in ER+ breast cancer patients on antiestrogenic medication?

2 . Is the addition of only 4 minutes of daily isometric exercises to this high protein diet more effective to improve their body composition?

3. How does the surgery, chemotherapy and antiestrogenic medication typeinfluences the effects of these interventions?

\section{Study Design}

- duration: 12 months

- number of patients: 165

- inclusion criteria:

- $\mathrm{ER}+/ \mathrm{PR} \pm / \mathrm{HER} 2$-luminal A and B breast cancer patients after surgery and chemoterapy, on antiestrogenic medication.

- Overweight

- exclusion criteria:

- Diabetes, thyroid or renal disease, eating disorders, depression, osteoporosis.

- interventions (Table 1):

- Diet group - D,

- Diet combined with 4 minutes of isometric exercises - D+Ex.

- monitoring:

- Body measurements were taken on a multi-frequency bioelectrical scale: total body weight (W), body fat percentage $(\% \mathrm{BF})$ and visceral fat percentage (\% VF)

- Food journal: patients were instructed

Table 1. Initial distribution of the patients

\begin{tabular}{llccc}
\hline & & & Intervention & \\
\hline & & Diet & Diet + exercises & Total \\
\hline Sample size & Initial & 83 & 82 & 165 \\
& 6 months & 64 & 71 & 135 \\
& 12 months & 40 & 41 & 81 \\
\hline \multirow{2}{*}{ Type of surgery } & Mastectomy & 58 & 62 & 120 \\
& Conservatory & 25 & 20 & 45 \\
\hline Type of chemotherapy & A & 28 & 20 & 48 \\
& NA & 30 & 30 & 60 \\
& NA\&A & 13 & 25 & 38 \\
& no chemotherapy & 12 & 7 & 19 \\
\hline Type of antiestrogenic & Aromatase inhibitors & 44 & 48 & 92 \\
& Tamoxifen & 39 & 34 & 73 \\
\hline
\end{tabular}


to keep a daily food log where to write the time they took each meal, exactly what it contained and in what quantity and if they were hungry or not when they ate.

\section{Interventions}

A high protein diet based on foods naturally high in proteins, omega-3 fatty acids, calcium, pro- $^{-}$and prebiotics can improve body composition by increasing insulin and leptin sensitivity, ameliorating dysbiosis and counteracting skeletal muscle protein catabolism. Additionally, it can assist in recurrence prevention through a moderate intake of glucose.

Patients were given a table on which foods were classified as proteins, carbohydrates or fibres supplying sources and were taught to consume them at each meal. Toprevent sarcopenia and to counteract the Warburg effect (especially in patients during neoadjuvant chemotherapy) we decreased the recommended percentage of carbohydrate intake from the common $55-60 \%$ to only $40 \%$. Protein intake was calculated to reach $1.5 \mathrm{~g} / \mathrm{kg}$ body, which practically meant a 25-30 g protein intake per meal for most patients. Also, current scientific literature does not support a low-fat approach for ER+ breast cancer patients, thus we recommended a $25-30 \%$ fat intake from foods sources of omega-3 fatty acids (fish, olive oil, raw nuts and seeds) and medium chain triglycerides mainly from fermented dairy foods (yoghurt, sour milk and kephir).

To prevent anaemia, we instructed them to eat foods high in proteins and calcium such as yoghurt, sour milk and kephir, raw seeds and nutsat different meals than foods high in iron just like fish, chicken, eggs, beans, chickpeas and other lentils. To prevent dysbiosis we instructed them to vary the food they eat as much as possible from day to day, to avoid eating foods containing unpasteurized raw animal ingredients (like unpasteurized ice cream or mayonnaise, sauces, deli meats or cheese, smoked raw fish, canned fish or roe), and to eat at least two fermented dairies portions per day.
To improve eating behaviour, we explained the metabolic differences between eating when not hungry and eating when physically hungry and we asked patients to learn to recognize gastric hunger and to respect it by not eating when not feeling hungry and by eating within a maximum of 1 hour after feeling this way (25).

To sustain an effective lipolysis, beta-oxidation and complete fatty acids catabolism for energy, when not hungry patients could only drink plain water when not feeling hungry and they were asked not to consume snacks, and other drinks. One coffee was allowed at the first meal of the day, and tea with other meals. However, they were not allowed in between meals due to caffeine and theine impact on insulin secretion. In addition, no soft drinks were allowed due to their impact on presynaptic dopamine re-transporters and on hypothalamic leptin sensitivity.

Also, to ensure a proper gastric emptying time, an interval of 2 hours' minimum was recommended between taking any meal and sleeping. And, to avoid phytoestrogen interaction with antiestrogenic treatments, we recommended the complete avoidance of plant supplements and we asked patients to only take vitamins and minerals at their oncologists' recommendation.

As for the isometric exercises, patients were taught how to perform 7 of them, one for each day of the week. All 7 exercises involved maintaining whole body balance for 1 minute, four times per day.

The authors certify that they comply with the ethical guidelines for authorship and publishing of this article. The investigators obtained informed consent from each study participant. The study has been performed in accordance with the ethical standards laid down in the 1964 Declaration of Helsinki and its later amendments. The authors declare no conflict of interest.

\section{Statistical Analysis}

Results were initially analysed using a Friedman Test, then Post hoc analysis with 
Wilcoxon signed-rank tests were conducted if Friedman Test was significant, with a Bonferroni correction, resulting in a significance level set at $p<0.017$. Friedman Test and Wilcoxon signed-rank tests were conducted using the exact tests and for Wilcoxon signedrank tests one-tailed significance was used.

\section{Results}

The patients' 1-year results show that both $\mathrm{D}+\mathrm{Ex}$ and $\mathrm{D}$ patients obtained statistically significant weight loss and fat loss, but D patients did not obtain statistical significant fat loss throughout the entire duration of the intervention. When comparing initial values with the ones measured at 12 th months, D patients lost 3,3 kg, 3,2\% $\mathrm{BF}$ and $1 \%$ visceral fat, while D+Ex patients lost $6,5 \mathrm{~kg}, 3,3 \% \mathrm{BF}$ and $2 \%$ visceral fat (Table 2 ).

The D+Ex patients had a significant loss of total body weight $\left(\chi^{2}(2)=56.54, \mathrm{p}<.05\right)$, of body fat $\left(\chi^{2}(2)=38.02, p<.05\right)$ and of visceral fat $\left(\chi^{2}(2)=38.15, \mathrm{p}<.05\right)$.

Wilcoxon tests were used to follow up these findings, with a Bonferroni correction, resulting a .017 level of significance. The weight significantly changed from the start to 6 months $(\mathrm{Z}=-5.45, \mathrm{p}=.000)$, from 6 months to 12 months $(\mathrm{Z}=-4.47, \mathrm{p}=.000)$ and from start to 12 months $(\mathrm{Z}=-5.51, \mathrm{p}=.000)$. The body fat percentage also changed significantly from the start to 6 months $(\mathrm{Z}=-4.50, \mathrm{p}=.000)$, from 6 months to 12 months $(\mathrm{Z}=-3.56, \mathrm{p}=.000)$ and from start to 12 months $(Z=-5.16, p=.000)$. It was the same case with the visceral fat percentage, with a significant change from the start to 6 months ( $Z$ $=-4.63, p=.000)$, from 6 months to 12 months $(\mathrm{Z}=-2.78, \mathrm{p}=.002)$ and from start to 12 months $(\mathrm{Z}=-4.61, \mathrm{p}=.000)$.

The results for the $\mathrm{D}$ patients show a significant loss of total body weight $\left(\chi^{2}(2)=\right.$ $35.94, p<.05)$, of body fat $\left(\chi^{2}(2)=10.20, p<.05\right)$ and of visceral fat $\left(\chi^{2}(2)=18.33, p<.05\right)$. The following up Wilcoxon tests show that only the weight loss was significant from start to 6 months ( $\mathrm{Z}=-4.38, \mathrm{p}=.000)$, from 6 months to 12 months $(\mathrm{Z}=-2.18, \mathrm{p}=.014)$ and from start to 12 months $(\mathrm{Z}=-4.58, \mathrm{p}=.000)$.

The body fat percentage did not significantly

Table 2. Comparative 1-year results for weightand adiposity correlated with the type of intervention

\begin{tabular}{|c|c|c|c|c|c|c|c|c|c|}
\hline \multirow[t]{2}{*}{ Intervention } & \multirow[t]{2}{*}{ Measurements } & \multirow[t]{2}{*}{ Mean } & \multirow[t]{2}{*}{ SD } & \multirow[t]{2}{*}{ Min } & \multirow[t]{2}{*}{$\operatorname{Max}$} & \multicolumn{3}{|c|}{ Percentiles } & \multirow[t]{2}{*}{ p } \\
\hline & & & & & & $25^{\text {th }}$ & $50^{\text {th }}$ (Median) & $75^{\text {th }}$ & \\
\hline \multirow{9}{*}{$\begin{array}{c}\text { Diet } \\
(n=40)\end{array}$} & Initial W (kg) & 74.55 & 14.15 & 58.8 & 127.0 & 65.77 & 70.00 & 80.15 & \\
\hline & $6^{\text {th }}$ month W $(\mathrm{kg})$ & 71.62 & 13.89 & 54.6 & 120.1 & 63.25 & 67.70 & 76.00 & $.000^{\mathrm{a}}$ \\
\hline & $12^{\text {th }}$ month W $(\mathrm{kg})$ & 70.88 & 13.93 & 52.5 & 117.0 & 61.35 & 66.65 & 76.67 & $.014^{b}$ \\
\hline & Initial BF\% & 39.19 & 5.46 & 30.0 & 56.6 & 34.75 & 39.15 & 41.73 & \\
\hline & $6^{\text {th }}$ month $\mathrm{BF} \%$ & 38.37 & 6.41 & 26.9 & 53.0 & 33.25 & 37.55 & 44.40 & $.021^{\mathrm{a}}$ \\
\hline & $12^{\text {th }}$ month BF\% & 37.33 & 6.36 & 26.1 & 51.2 & 31.80 & 35.95 & 42.60 & $.008^{b}$ \\
\hline & Initial VF\% & 8.43 & 2.33 & 4 & 14 & 7.00 & 8.00 & 10.00 & \\
\hline & $6^{\text {th }}$ month VF\% & 7.85 & 2.27 & 3 & 13 & 6.00 & 8.00 & 9.00 & $.001^{\mathrm{a}}$ \\
\hline & $12^{\text {th }}$ month VF\% & 7.55 & 2.24 & 4 & 12 & 6.00 & 7.00 & 9.00 & $.029^{b}$ \\
\hline \multirow{9}{*}{$\begin{array}{l}\text { Diet }+ \text { Exercise } \\
(n=41)\end{array}$} & Initial W (kg) & 79.00 & 14.83 & 58.3 & 109.4 & 63.80 & 80.80 & 88.85 & \\
\hline & $6^{\text {th }}$ month W $(\mathrm{kg})$ & 74.52 & 13.25 & 54.3 & 100.0 & 61.60 & 75.40 & 85.30 & $.000^{\mathrm{a}}$ \\
\hline & $12^{\text {th }}$ month W $(\mathrm{kg})$ & 72.66 & 12.69 & 52.1 & 97.9 & 62.20 & 74.30 & 82.40 & $.000^{b}$ \\
\hline & Initial BF\% & 41.30 & 5.41 & 30.0 & 55.8 & 37.00 & 40.40 & 46.10 & \\
\hline & $6^{\text {th }}$ month BF\% & 37.95 & 5.95 & 25.9 & 50.6 & 34.65 & 36.70 & 43.25 & $.000^{\mathrm{a}}$ \\
\hline & $12^{\text {th }}$ month BF\% & 36.40 & 6.21 & 25.3 & 51.8 & 31.85 & 37.10 & 40.75 & $.000^{\mathrm{b}}$ \\
\hline & Initial VF\% & 9.98 & 2.54 & 5 & 15 & 8.00 & 10.00 & 12.00 & \\
\hline & $6^{\text {th }}$ month VF\% & 8.63 & 2.38 & 4 & 13 & 7.00 & 9.00 & 11.00 & $.000^{\mathrm{a}}$ \\
\hline & $12^{\text {th }}$ month VF\% & 8.15 & 2.55 & 4 & 13 & 6.00 & 8.00 & 10.00 & $.002^{b}$ \\
\hline
\end{tabular}

$\mathrm{n}=$ no. of patients following the intervention at 12 months; $\mathrm{W}=$ weight, $\mathrm{BF}=$ body fat, VF = visceral fat; $\mathrm{a}=$ Wilcoxon Signed Ranks Test $p$-value for measurement between initial and the $6^{\text {th }}$ month; $b=$ Wilcoxon Signed Ranks Test $p$-value for measurement between the $6^{\text {th }}$ and the $12^{\text {th }}$ month; $p$-values $<0.017$ are significant (marked in grey) 
decrease from start to 6 months $(\mathrm{Z}=-2.02, \mathrm{p}=$ .021) and it did decrease significantly from 6 months to 12 months $(\mathrm{Z}=-2.41, \mathrm{p}=.008)$ and from start to 12 months $(\mathrm{Z}=-2.56, \mathrm{p}=.005)$.

The loss of visceral fat percentage was significant from start to 6 months $(\mathrm{Z}=-3.20$, $\mathrm{p}=.001)$, and to 12 months $(\mathrm{Z}=-3.75, \mathrm{p}=.000)$, but not from 6 months to 12 months $(\mathrm{Z}=-2.01$, $\mathrm{p}=.029)$.
Correlating these patients' 1 -year results with the type of surgery, we concluded that patients with mastectomy obtained statistically significant weight and fat loss, while patients with conservatory treatment obtained statistically significant results at the $12^{\text {th }}$ month weight and adiposity measurement only in the D+Ex intervention (Table 3).

Patients with D+Ex intervention obtained

Table 3. Comparative 1-year results for weightand adiposity correlated with the type of surgery

\begin{tabular}{|c|c|c|c|c|c|c|c|c|c|c|}
\hline \multirow[t]{2}{*}{ Int. } & \multirow[t]{2}{*}{ Surgery type } & \multirow[t]{2}{*}{ Measurements } & \multirow[t]{2}{*}{ Mean } & \multirow[t]{2}{*}{ SD } & \multirow[t]{2}{*}{ Min } & \multirow[t]{2}{*}{ Max } & \multicolumn{3}{|c|}{ Percentiles } & \multirow[t]{2}{*}{$p$} \\
\hline & & & & & & & $25^{\text {th }}$ & $50^{\text {th }}$ (Median) & $75^{\text {th }}$ & \\
\hline \multirow[t]{18}{*}{ Diet } & Mastectomy & Initial W (kg) & 73.73 & 13.74 & 58.80 & 127.00 & 65.78 & 70.00 & 77.45 & \\
\hline & $(n=28)$ & $6^{\text {th }}$ month W $(\mathrm{kg})$ & 70.74 & 13.38 & 54.60 & 120.10 & 63.28 & 67.70 & 74.83 & $.000^{\circ}$ \\
\hline & & $12^{\text {th }}$ month W $(\mathrm{kg})$ & 69.46 & 13.32 & 52.50 & 117.00 & 60.35 & 66.65 & 74.33 & .001 \\
\hline & & Initial BF\% & 39.41 & 5.65 & 30.80 & 56.60 & 34.45 & 39.80 & 41.65 & \\
\hline & & $6^{\text {th }}$ month $B F \%$ & 38.63 & 6.28 & 29.70 & 53.00 & 33.35 & 37.35 & 43.90 & $.046^{\circ}$ \\
\hline & & $12^{\text {th }}$ month BF\% & 37.35 & 6.22 & 27.50 & 51.20 & 31.70 & 36.95 & 41.35 & .002 \\
\hline & & Initial VF\% & 8.46 & 2.38 & 4.00 & 13.00 & 6.25 & 8.00 & 10.00 & \\
\hline & & $6^{\text {th }}$ month VF\% & 7.89 & 2.13 & 5.00 & 12.00 & 6.25 & 7.50 & 8.75 & $.005^{\circ}$ \\
\hline & & $12^{\text {th }}$ month VF\% & 7.50 & 2.27 & 4.00 & 12.00 & 6.00 & 7.00 & 9.00 & .021 \\
\hline & Conservatory & Initial W (kg) & 76.46 & 15.52 & 61.60 & 114.60 & 64.55 & 70.95 & 86.88 & \\
\hline & treatment & $6^{\text {th }}$ month W $(\mathrm{kg})$ & 73.69 & 15.45 & 58.60 & 112.40 & 62.20 & 68.00 & 84.28 & - \\
\hline & $(n=12)$ & $12^{\text {th }}$ month W $(\mathrm{kg})$ & 74.21 & 15.35 & 59.90 & 110.80 & 62.98 & 69.05 & 85.65 & - \\
\hline & & Initial BF\% & 38.68 & 5.20 & 30.00 & 47.70 & 35.28 & 38.30 & 42.40 & \\
\hline & & $6^{\text {th }}$ month $\mathrm{BF} \%$ & 37.78 & 6.96 & 26.90 & 47.70 & 32.73 & 37.60 & 44.40 & - \\
\hline & & $12^{\text {th }}$ month BF\% & 37.30 & 6.97 & 26.10 & 49.10 & 32.13 & 34.85 & 43.23 & - \\
\hline & & Initial VF\% & 8.33 & 2.31 & 5.00 & 14.00 & 7.00 & 8.00 & 9.75 & \\
\hline & & $6^{\text {th }}$ month VF\% & 7.75 & 2.67 & 3.00 & 13.00 & 6.00 & 8.00 & 9.00 & - \\
\hline & & $12^{\text {th }}$ month VF\% & 7.67 & 2.27 & 5.00 & 12.00 & 5.25 & 8.00 & 8.75 & - \\
\hline \multirow{18}{*}{ Diet+Ex } & Mastectomy & Initial W (kg) & 78.92 & 14.89 & 58.30 & 109.40 & 63.80 & 80.80 & 88.80 & \\
\hline & $(n=35)$ & $6^{\text {th }}$ month W $(\mathrm{kg})$ & 74.35 & 12.92 & 54.30 & 100.00 & 61.60 & 75.40 & 84.90 & $.000^{\circ}$ \\
\hline & & $12^{\text {th }}$ month $W(\mathrm{~kg})$ & 72.63 & 12.41 & 52.10 & 97.90 & 62.40 & 74.30 & 82.10 & $.000^{\prime}$ \\
\hline & & Initial BF\% & 41.46 & 5.75 & 30.00 & 55.80 & 36.60 & 40.40 & 46.20 & \\
\hline & & $6^{\text {th }}$ month BF\% & 37.90 & 6.22 & 25.90 & 50.60 & 33.60 & 36.70 & 43.80 & $.000^{\circ}$ \\
\hline & & $12^{\text {th }}$ month BF\% & 36.56 & 6.48 & 25.30 & 51.80 & 31.60 & 37.10 & 40.80 & .001 \\
\hline & & Initial VF\% & 9.94 & 2.61 & 5.00 & 15.00 & 7.00 & 10.00 & 12.00 & \\
\hline & & $6^{\text {th }}$ month VF\% & 8.63 & 2.47 & 4.00 & 13.00 & 7.00 & 9.00 & 11.00 & $.000^{\circ}$ \\
\hline & & $12^{\text {th }}$ month VF\% & 8.23 & 2.62 & 4.00 & 13.00 & 6.00 & 8.00 & 10.00 & .013 \\
\hline & Conservatory & Initial W (kg) & 79.47 & 15.88 & 63.50 & 99.40 & 64.40 & 76.65 & 96.78 & \\
\hline & treatment & $6^{\text {th }}$ month W $(\mathrm{kg})$ & 75.58 & 16.39 & 58.50 & 94.10 & 59.10 & 74.05 & 93.65 & .015 \\
\hline & $(n=6)$ & $12^{\text {th }}$ month W $(\mathrm{kg})$ & 72.83 & 15.56 & 57.10 & 91.10 & 57.18 & 71.15 & 89.75 & .015 \\
\hline & & Initial BF\% & 40.42 & 2.94 & 37.20 & 43.60 & 37.43 & 40.50 & 43.30 & \\
\hline & & $6^{\text {th }}$ month BF\% & 38.27 & 4.56 & 35.00 & 47.00 & 35.30 & 36.40 & 41.30 & .109 \\
\hline & & $12^{\text {th }}$ month BF\% & 35.50 & 4.78 & 30.30 & 44.20 & 32.55 & 34.05 & 38.88 & .015 \\
\hline & & Initial VF\% & 10.17 & 2.32 & 8.00 & 14.00 & 8.00 & 10.00 & 11.75 & \\
\hline & & $6^{\text {th }}$ month VF\% & 8.67 & 1.97 & 6.00 & 11.00 & 6.75 & 9.00 & 10.25 & $.063^{\circ}$ \\
\hline & & $12^{\text {th }}$ month VF\% & 7.67 & 2.16 & 5.00 & 11.00 & 5.75 & 7.50 & 9.50 & .031 \\
\hline
\end{tabular}

$\mathrm{n}=$ no. of patients following the intervention at 12 months; $\mathrm{W}=$ weight, $\mathrm{BF}=$ body fat, $\mathrm{VF}=$ visceral fat; $\mathrm{a}=$ Wilcoxon Signed Ranks Test $p$-value for measurement between initial and the 6 th month; $b=$ Wilcoxon Signed Ranks Test $p$-value for measurement between the $6^{\text {th }}$ and the $12^{\text {th }}$ month; p-values $<0.017$ are significant (marked in grey); - initial Friedman Test not statistically significant, no post hoc tests run 
Table 4. Comparative 1-year results for weight and adiposity correlated with the type of chemotherapy

\begin{tabular}{|c|c|c|c|c|c|c|c|c|c|c|}
\hline \multirow[t]{2}{*}{ Int. } & \multirow[t]{2}{*}{ CH type } & \multirow[t]{2}{*}{ Measurements } & \multirow[t]{2}{*}{ Mean } & \multirow[t]{2}{*}{ SD } & \multirow[t]{2}{*}{ Min } & \multirow[t]{2}{*}{ Max } & \multicolumn{3}{|c|}{ Percentiles } & \multirow[t]{2}{*}{ p } \\
\hline & & & & & & & $25^{\text {th }}$ & $50^{\text {th }}$ (Median) & $75^{\text {th }}$ & \\
\hline \multirow[t]{27}{*}{ Diet } & $N A(n=13)$ & Initial W (kg) & 70.00 & 8.23 & 91.90 & 91.90 & 64.30 & 67.20 & 72.30 & \\
\hline & & $6^{\text {th }}$ month W $(\mathrm{kg})$ & 66.89 & 8.57 & 87.70 & 87.70 & 60.65 & 64.10 & 70.70 & $.011^{\mathrm{a}}$ \\
\hline & & $12^{\text {th }}$ month W $(\mathrm{kg})$ & 66.13 & 9.08 & 88.40 & 88.40 & 60.60 & 62.90 & 71.95 & $.081^{b}$ \\
\hline & & Initial BF\% & 36.45 & 4.11 & 30.00 & 41.80 & 33.25 & 36.10 & 40.55 & \\
\hline & & $6^{\text {th }}$ month BF\% & 35.83 & 5.92 & 27.80 & 47.60 & 31.20 & 34.70 & 40.05 & - \\
\hline & & $12^{\text {th }}$ month BF\% & 34.38 & 4.98 & 28.70 & 45.30 & 31.25 & 32.10 & 36.80 & - \\
\hline & & Initial VF\% & 7.77 & 2.05 & 5.00 & 13.00 & 6.00 & 8.00 & 8.00 & \\
\hline & & $6^{\text {th }}$ month VF\% & 7.15 & 2.03 & 3.00 & 12.00 & 6.00 & 7.00 & 8.00 & $.017^{\mathrm{a}}$ \\
\hline & & $12^{\text {th }}$ month VF\% & 6.77 & 1.83 & 5.00 & 12.00 & 6.00 & 6.00 & 7.00 & $.117^{\mathrm{b}}$ \\
\hline & $A(n=17)$ & Initial W (kg) & 78.99 & 18.36 & 61.60 & 127.00 & 66.60 & 72.20 & 88.25 & \\
\hline & & $6^{\text {th }}$ month W $(\mathrm{kg})$ & 76.11 & 17.69 & 54.60 & 120.10 & 64.80 & 70.20 & 86.90 & $.004^{\mathrm{a}}$ \\
\hline & & $12^{\text {th }}$ month W $(\mathrm{kg})$ & 75.44 & 17.91 & 52.50 & 117.00 & 63.85 & 70.60 & 87.90 & $.105^{b}$ \\
\hline & & Initial BF\% & 42.18 & 5.76 & 33.40 & 56.60 & 37.55 & 41.10 & 46.35 & \\
\hline & & $6^{\text {th }}$ month BF\% & 40.97 & 6.01 & 30.00 & 53.00 & 37.00 & 40.10 & 45.80 & - \\
\hline & & $12^{\text {th }}$ month BF\% & 40.39 & 6.78 & 27.50 & 51.20 & 34.05 & 41.20 & 46.00 & - \\
\hline & & Initial VF\% & 9.29 & 2.05 & 6.00 & 14.00 & 8.00 & 9.00 & 10.50 & \\
\hline & & $6^{\text {th }}$ month VF\% & 8.71 & 2.20 & 5.00 & 13.00 & 7.50 & 8.00 & 10.50 & - \\
\hline & & $12^{\text {th }}$ month VF\% & 8.47 & 2.26 & 5.00 & 12.00 & 7.00 & 8.00 & 10.00 & - \\
\hline & $N A+A(n=6)$ & Initial W (kg) & 71.36 & 11.59 & 58.80 & 89.60 & 59.10 & 72.35 & 79.33 & \\
\hline & & $6^{\text {th }}$ month W $(\mathrm{kg})$ & 68.86 & 11.41 & 54.70 & 86.50 & 58.82 & 68.05 & 78.40 & $.047^{\mathrm{a}}$ \\
\hline & & $12^{\text {th }}$ month W $(\mathrm{kg})$ & 68.08 & 10.30 & 55.20 & 83.20 & 58.05 & 67.85 & 77.35 & $.219^{b}$ \\
\hline & & Initial BF\% & 38.50 & 4.65 & 33.70 & 46.10 & 34.00 & 37.95 & 42.43 & \\
\hline & & $6^{\text {th }}$ month $\mathrm{BF} \%$ & 39.97 & 6.51 & 32.10 & 48.60 & 32.78 & 40.70 & 45.68 & - \\
\hline & & $12^{\text {th }}$ month BF\% & 37.95 & 4.65 & 31.80 & 43.20 & 32.48 & 39.30 & 41.85 & - \\
\hline & & Initial VF\% & 8.00 & 3.46 & 4.00 & 13.00 & 4.75 & 7.50 & 11.50 & \\
\hline & & $6^{\text {th }}$ month VF\% & 7.67 & 2.87 & 5.00 & 12.00 & 5.00 & 7.00 & 10.50 & - \\
\hline & & $12^{\text {th }}$ month VF\% & 7.17 & 2.85 & 4.00 & 11.00 & 4.75 & 7.00 & 9.50 & - \\
\hline \multirow[t]{27}{*}{ Diet + Ex } & $N A(n=18)$ & Initial W (kg) & 81.21 & 14.29 & 61.00 & 106.00 & 64.78 & 85.15 & 91.40 & \\
\hline & & $6^{\text {th }}$ month W $(\mathrm{kg})$ & 75.09 & 12.10 & 58.50 & 94.10 & 61.60 & 76.70 & 84.48 & $.000^{\mathrm{a}}$ \\
\hline & & $12^{\text {th }}$ month $\mathrm{W}(\mathrm{kg})$ & 73.19 & 11.14 & 57.20 & 91.10 & 62.40 & 74.85 & 81.20 & $.001^{\mathrm{b}}$ \\
\hline & & Initial BF\% & 42.17 & 5.33 & 34.90 & 55.80 & 37.45 & 41.30 & 45.83 & \\
\hline & & $6^{\text {th }}$ month BF\% & 38.34 & 5.70 & 28.70 & 50.60 & 34.78 & 38.20 & 43.05 & $.001^{\mathrm{a}}$ \\
\hline & & $12^{\text {th }}$ month BF\% & 36.10 & 6.38 & 26.10 & 51.80 & 30.18 & 36.75 & 39.43 & $.001^{b}$ \\
\hline & & Initial VF\% & 10.72 & 2.76 & 6.00 & 15.00 & 8.50 & 11.00 & 12.25 & \\
\hline & & $6^{\text {th }}$ month VF\% & 8.83 & 2.60 & 4.00 & 13.00 & 6.00 & 9.50 & 10.25 & $.000^{\mathrm{a}}$ \\
\hline & & $12^{\text {th }}$ month VF\% & 8.17 & 2.62 & 4.00 & 13.00 & 6.00 & 8.00 & 10.25 & $.016^{\mathrm{b}}$ \\
\hline & $A(n=7)$ & Initial W (kg) & 78.39 & 16.37 & 63.50 & 109.40 & 63.80 & 73.60 & 88.60 & \\
\hline & & $6^{\text {th }}$ month W $(\mathrm{kg})$ & 74.56 & 14.18 & 59.30 & 99.00 & 61.90 & 71.20 & 85.70 & $.008^{a}$ \\
\hline & & $12^{\text {th }}$ month W $(\mathrm{kg})$ & 71.90 & 13.13 & 57.10 & 93.80 & 60.30 & 69.80 & 82.70 & $.008^{b}$ \\
\hline & & Initial BF\% & 40.37 & 4.27 & 35.50 & 47.70 & 37.20 & 38.70 & 43.20 & \\
\hline & & $6^{\text {th }}$ month BF\% & 37.53 & 3.93 & 34.70 & 45.40 & 35.00 & 35.40 & 40.10 & $.015^{\mathrm{a}}$ \\
\hline & & $12^{\text {th }}$ month BF\% & 35.93 & 4.86 & 32.10 & 45.60 & 32.30 & 34.00 & 39.00 & $.015^{b}$ \\
\hline & & Initial VF\% & 9.86 & 2.41 & 7.00 & 14.00 & 8.00 & 10.00 & 11.00 & \\
\hline & & $6^{\text {th }}$ month VF\% & 8.86 & 2.04 & 7.00 & 11.00 & 7.00 & 8.00 & 11.00 & $.125^{\mathrm{a}}$ \\
\hline & & $12^{\text {th }}$ month VF\% & 7.86 & 2.12 & 5.00 & 10.00 & 6.00 & 7.00 & 10.00 & $.015^{\mathrm{b}}$ \\
\hline & $N A+A(n=13)$ & Initial W (kg) & 75.67 & 14.35 & 59.80 & 102.20 & 61.75 & 72.80 & 87.75 & \\
\hline & & $6^{\text {th }}$ month W $(\mathrm{kg})$ & 72.52 & 13.81 & 54.30 & 92.70 & 58.25 & 71.00 & 85.60 & $.001^{\mathrm{a}}$ \\
\hline & & $12^{\text {th }}$ month $W(\mathrm{~kg})$ & 71.30 & 13.81 & 52.10 & 89.30 & 56.40 & 71.50 & 82.65 & $.057^{b}$ \\
\hline & & Initial BF\% & 40.72 & 5.81 & 30.00 & 49.50 & 36.55 & 40.40 & 46.15 & \\
\hline & & $6^{\text {th }}$ month BF\% & 37.32 & 6.98 & 25.90 & 49.30 & 32.80 & 35.00 & 44.55 & $.000^{\mathrm{a}}$ \\
\hline & & $12^{\text {th }}$ month BF\% & 36.85 & 6.71 & 25.30 & 49.80 & 32.55 & 37.40 & 41.20 & $.271^{b}$ \\
\hline & & Initial VF\% & 8.92 & 2.06 & 5.00 & 12.00 & 7.50 & 9.00 & 10.50 & \\
\hline & & $6^{\text {th }}$ month VF\% & 7.92 & 2.25 & 4.00 & 12.00 & 6.50 & 7.00 & 10.00 & $.004^{\mathrm{a}}$ \\
\hline & & $12^{\text {th }}$ month VF\% & 7.85 & 2.73 & 4.00 & 13.00 & 5.50 & 7.00 & 10.00 & $.249^{b}$ \\
\hline
\end{tabular}

$\mathrm{n}=$ no. of patients following the intervention at 12 months; $\mathrm{W}=$ weight, $\mathrm{BF}=$ body fat, VF = visceral fat; a $=$ Wilcoxon Signed Ranks Test $p$-value for measurement between initial and the 6th month; $b=$ Wilcoxon Signed Ranks Test $p$-value for measurement between the $6^{\text {th }}$ and the $12^{\text {th }}$ month; p-values $<0.017$ are significant (marked in grey); - initial Friedman Test not statistically significant, no post hoc tests run; $\mathrm{CH}=$ chemotherapy, $\mathrm{NA}=$ neoadjuvant, $\mathrm{A}=$ adjuvant, $\mathrm{NA}+\mathrm{A}=$ both neoadjuvant and adjuvant chemotherapy 
statistically significant weight and fat loss regardless of chemotherapy type, while patients with $\mathrm{D}$ intervention lost weight and did not lost body fat when administered adjuvant or both neoadjuvant and adjuvant chemotherapy. D Patients with neoadjuvant chemotherapy alone obtained both weight loss and visceral fat loss, but they did not obtain body fat loss (Table 4).

Patients on AI obtained statistically significant results for both weight and fat loss regardless of the intervention they were assigned to. And patients on Tamoxifen with the D intervention only lost weight, while those on the D+EX intervention lost weight and body and visceral fat (Table 5).

Table 5. Comparative 1-year results for weight and adiposity correlated with the type of antiestrogenic treatment (AET)

\begin{tabular}{|c|c|c|c|c|c|c|c|c|c|c|}
\hline \multirow[t]{2}{*}{ Int. } & \multirow[t]{2}{*}{ AET type } & \multirow[t]{2}{*}{ Measurements } & \multirow[t]{2}{*}{ Mean } & \multirow[t]{2}{*}{ SD } & \multirow[t]{2}{*}{ Min } & \multirow[t]{2}{*}{ Max } & \multicolumn{3}{|c|}{ Percentiles } & \multirow[t]{2}{*}{ p } \\
\hline & & & & & & & $25^{\text {th }}$ & $50^{\text {th }}$ (Median) & $75^{\text {th }}$ & \\
\hline \multirow[t]{18}{*}{ Diet } & Tamoxifen $(n=15)$ & Initial W (kg) & 77.98 & 19.35 & 63.00 & 127.00 & 65.70 & 70.00 & 87.80 & \\
\hline & & $6^{\text {th }}$ month $\mathrm{W}(\mathrm{kg})$ & 75.33 & 18.59 & 58.60 & 120.10 & 63.80 & 67.80 & 86.80 & $.017^{\mathrm{a}}$ \\
\hline & & $12^{\text {th }}$ month $W(\mathrm{~kg})$ & 74.72 & 18.18 & 59.80 & 117.00 & 61.50 & 68.40 & 85.60 & $.025^{\mathrm{b}}$ \\
\hline & & Initial BF\% & 40.22 & 7.30 & 30.00 & 56.60 & 34.60 & 38.10 & 47.00 & \\
\hline & & $6^{\text {th }}$ month BF\% & 38.95 & 7.44 & 27.80 & 53.00 & 33.40 & 37.20 & 46.20 & - \\
\hline & & $12^{\text {th }}$ month $\mathrm{BF} \%$ & 38.85 & 7.24 & 27.50 & 51.20 & 31.80 & 39.70 & 45.70 & - \\
\hline & & Initial VF\% & 8.53 & 2.53 & 5.00 & 14.00 & 7.00 & 8.00 & 10.00 & \\
\hline & & $6^{\text {th }}$ month VF\% & 7.93 & 2.63 & 3.00 & 13.00 & 6.00 & 8.00 & 8.00 & - \\
\hline & & $12^{\text {th }}$ month VF\% & 7.80 & 2.51 & 5.00 & 12.00 & 6.00 & 7.00 & 9.00 & - \\
\hline & $\mathrm{Al}(\mathrm{n}=25)$ & Initial W (kg) & 72.49 & 9.75 & 58.80 & 91.90 & 65.20 & 70.00 & 78.50 & \\
\hline & & $6^{\text {th }}$ month W $(\mathrm{kg})$ & 69.40 & 9.91 & 54.60 & 87.70 & 62.40 & 67.60 & 75.90 & $.000^{\mathrm{a}}$ \\
\hline & & $12^{\text {th }}$ month $\mathrm{W}(\mathrm{kg})$ & 68.59 & 10.40 & 52.50 & 90.20 & 60.00 & 66.00 & 76.25 & $.018^{\mathrm{b}}$ \\
\hline & & Initial BF\% & 38.57 & 4.04 & 30.80 & 46.10 & 34.80 & 39.80 & 41.35 & \\
\hline & & $6^{\text {th }}$ month BF\% & 38.03 & 5.84 & 26.90 & 48.60 & 32.85 & 37.60 & 43.10 & $.155^{\mathrm{a}}$ \\
\hline & & $12^{\text {th }}$ month BF\% & 36.42 & 5.74 & 26.10 & 49.10 & 31.75 & 35.80 & 41.30 & $.001^{\mathrm{b}}$ \\
\hline & & Initial VF\% & 8.36 & 2.25 & 4.00 & 13.00 & 6.50 & 8.00 & 10.00 & \\
\hline & & $6^{\text {th }}$ month VF\% & 7.80 & 2.08 & 5.00 & 12.00 & 6.00 & 8.00 & 9.00 & $.012^{\mathrm{a}}$ \\
\hline & & $12^{\text {th }}$ month VF\% & 7.40 & 2.10 & 4.00 & 12.00 & 6.00 & 7.00 & 9.00 & $.015^{\mathrm{b}}$ \\
\hline \multirow[t]{18}{*}{ Diet + Ex } & Tamoxifen $(n=15)$ & Initial W (kg) & 80.79 & 13.72 & 59.80 & 102.70 & 65.90 & 86.00 & 88.90 & \\
\hline & & $6^{\text {th }}$ month W $(\mathrm{kg})$ & 76.51 & 13.77 & 55.10 & 100.00 & 61.60 & 80.60 & 86.30 & $.000^{\mathrm{a}}$ \\
\hline & & $12^{\text {th }}$ month $\mathrm{W}(\mathrm{kg})$ & 74.79 & 13.50 & 54.30 & 97.90 & 62.40 & 79.20 & 83.20 & $.011^{\mathrm{b}}$ \\
\hline & & Initial BF\% & 40.82 & 5.72 & 30.00 & 49.50 & 36.00 & 40.30 & 46.20 & \\
\hline & & $6^{\text {th }}$ month BF\% & 36.97 & 6.67 & 25.90 & 49.30 & 32.10 & 35.40 & 42.70 & $.003^{\mathrm{a}}$ \\
\hline & & $12^{\text {th }}$ month $\mathrm{BF} \%$ & 34.92 & 6.81 & 25.30 & 49.80 & 29.40 & 35.20 & 40.70 & $.002^{b}$ \\
\hline & & Initial VF\% & 9.20 & 2.31 & 5.00 & 13.00 & 7.00 & 9.00 & 11.00 & \\
\hline & & $6^{\text {th }}$ month VF\% & 7.80 & 2.65 & 4.00 & 12.00 & 6.00 & 7.00 & 10.00 & $.000^{\mathrm{a}}$ \\
\hline & & $12^{\text {th }}$ month VF\% & 7.33 & 2.32 & 4.00 & 12.00 & 5.00 & 7.00 & 10.00 & $.065^{\mathrm{b}}$ \\
\hline & Al $(n=26)$ & Initial W (kg) & 77.97 & 15.61 & 58.30 & 109.40 & 63.73 & 76.60 & 89.08 & \\
\hline & & $6^{\text {th }}$ month W $(\mathrm{kg})$ & 73.38 & 13.08 & 54.30 & 99.00 & 61.58 & 73.10 & 82.20 & $.000^{\mathrm{a}}$ \\
\hline & & $12^{\text {th }}$ month $W(\mathrm{~kg})$ & 71.43 & 12.31 & 52.10 & 93.80 & 61.58 & 72.90 & 79.30 & $.000^{\mathrm{b}}$ \\
\hline & & Initial BF\% & 41.58 & 5.32 & 31.20 & 55.80 & 37.28 & 41.30 & 45.80 & \\
\hline & & $6^{\text {th }}$ month BF\% & 38.52 & 5.57 & 28.90 & 50.60 & 34.93 & 37.35 & 44.15 & $.000^{\mathrm{a}}$ \\
\hline & & $12^{\text {th }}$ month $\mathrm{BF} \%$ & 37.26 & 5.81 & 27.10 & 51.80 & 33.05 & 37.25 & 41.73 & $.010^{\mathrm{b}}$ \\
\hline & & Initial VF\% & 10.42 & 2.61 & 6.00 & 15.00 & 8.00 & 11.00 & 12.00 & \\
\hline & & $6^{\text {th }}$ month VF\% & 9.12 & 2.12 & 6.00 & 13.00 & 7.00 & 9.50 & 11.00 & $.000^{\mathrm{a}}$ \\
\hline & & $12^{\text {th }}$ month VF\% & 8.62 & 2.59 & 4.00 & 13.00 & 6.75 & 8.50 & 11.00 & $.012^{\mathrm{b}}$ \\
\hline
\end{tabular}

$\mathrm{n}=$ no. of patients following the intervention at 12 months; $\mathrm{W}=$ weight, $\mathrm{BF}=$ body fat, $\mathrm{VF}=$ visceral fat; $\mathrm{a}=$ Wilcoxon Signed Ranks Test $p$-value for measurement between initial and the $6^{\text {th }}$ month; $b=$ Wilcoxon Signed Ranks Test $p$-value for measurement between the $6^{\text {th }}$ and the $12^{\text {th }}$ month; $p$-values $<0.017$ are significant (marked in grey); - initial Friedman Test not statistically significant, no post hoc tests run; $\mathrm{Al}=$ aromatase inhibitors 


\section{Discussions}

Many studies prove that high protein diets are effective in counteracting sarcopenia. Campbell et all questioned the recommended $0.8 \mathrm{~g} / \mathrm{kg}$ per day dietary allowance for protein as inadequate for older people to maintain skeletal muscle (26). Then, in 2007, he co-authored Leidy's study proving that higher protein intake preserves lean mass and satiety during weight loss interventions (27). The patients in our study were also at risk for sarcopenia, thus we used a $1.5 \mathrm{~g}$ protein intake/ kg body weight per day.

Paddon-Jones et all proved that high protein diets may represent a viable intervention for individuals at risk of sarcopenia in their 2004 study, when they managed to maintain lean leg massin patientsduring 28 days bed rest. But they offset the catabolic response to prolonged inactivity with essential amino acids and carbohydrates supplemented to mixed meals offered every $5 \mathrm{~h}$ (28). We used foods naturally high in proteins and we instructed patients to eat only when hungry. Then, in 2009, he proposed a novel and specific dietary approach to prevent or sarcopenia, recommending clinicians to stress the importance of ingesting 25-30g of protein with each meal, a recommendation we also used in our study (29).

Other studies using high protein diets in breast cancer patients found them effective but most used resistance exercise to counteract sarcopenia.To our knowledge, this is the first study to evaluate weight and body composition change in breast cancer patients on antiestrogenic medication using a 4' whole body isometric exercise protocol.

A 2002 study authored by DemarkWahnefried et all yielded promising results in preventing chemotherapy-induced weight and body composition changes among young women who received adjuvant chemotherapy for breast cancer (30). They used a specialized program of strength training, aerobic activity and a healthful diet $(\leq 20 \%$ fat; fruit, vegetable and calcium-rich).

In Denmark-Wahnefried's study more than half of the patients approached for the intervention refused to participate because the exercises where performed in hospital settings, which raised the recommendation that weight gain preventing interventions would be more effective with a home-based approach. Our study was a home-based study, but because the kinetic intervention was done at home without monitoring, we have no way of being sure if the patients did the exercises or not.

To determine the effectiveness of weight loss intervention for breast cancer survivors, Playdon et al performed a systematic review of 15 weight loss studies in breast cancer survivors in 2013 (31). Successful interventions used dietary, physical activity, and behaviour modification components, yet there was insufficient evidence to identify the interventions that led to successful weight loss, or to determine the weight loss necessary to affect biomarkers linked to breast cancer prognosis. The main drawbacks of the studies in this systematic review were short duration, the small study sample sizes and lack of follow-up beyond a $6^{-}$ monthperiod. Our study also has a small sample size, but we assessed patients at 12 months. Still we had a1-year dropout rate of almost 50\%.

Based on the day to day clinical practice, we expected that patients with conservative surgery would have better results but - as a group - patients with conservative surgery did not validate our expectations. The studied oncology nutrition literature generally finds no correlation between the type of surgery and the patients ability and/or willingness to lose weight after breast cancer treatment. The better results of our patients with mastectomy either in $\mathrm{D}$ or in $\mathrm{D}+\mathrm{Ex}$ intervention group were probably due to better individual adherence to dietary rules and not to the type of surgery per se.

Current literature supports both the fact that breast cancer patients gain weight during chemotherapy (32) that adjuvant administration of chemotherapy may lead to greater weight gain (33) and that longer administration of this type of treatment may have a more detrimental metabolic impact (34). What our study proved is that nutrition interventions alone might not be sufficient to counteract 
sarcopenia during chemotherapy be it neoadjuvant, adjuvant or both and that a minimal exercise protocol should be practiced during this treatment.

The results of our patients are not in line with other studies that show that aromatase inhibitors have higher musculoskeletal side effects (35) as our patients on Tamoxifen only lost weight and not fat neither at 6 nor at the 12 moth measurements.

Finally, about the way we measured body composition, multi-frequency BIA measurements can be easily used to assess patients' weight and body composition in clinical settings when limiting biased results as much as possible by ensuring the hydration and feeding status of the patient at the time of the measurement, and by doing measurements in the same standard conditions (36).

\section{Conclusions}

In conclusion, in $\mathrm{ER}+/ \mathrm{PR} \pm / \mathrm{HER} 2-$ luminal $\mathrm{A}$ and $\mathrm{B}$ breast cancer patients on antiestrogenic medication this 1-year diet intervention was effective in counteracting treatment associated weight gain, potentially improving long-term prognosis. And adding at least a minimal exercise protocol to oncology nutrition intervention is essential during chemotherapy and antiestrogenic treatment.

\section{Acknowledgments}

This paper was co-financed from the European Social Fund, through the Sectorial Operational Program Human Resources Development 2007-2013, contract POSDRU/187/1.5/S/155463 "Supporting excellence in scientific interdisciplinary doctoral research in the economic, medical and social fields", coordinator The Bucharest University of Economic Studies.

\section{References}

1. Chlebowski RT, Aiello E, McTiernan A. Weight loss in breast cancer patient management. J Clin Oncol. 2002;20(4):1128-43.

2. McAuley KA, Hopkins CM, Smith KJ, McLay RT, Williams SM, Taylor RW, et al. Comparison of high-fat and high-protein diets with a high-carbohydrate diet in insulin-resistant obese women.
Diabetologia. 2005;48(1):8-16. Epub 2004 Dec 23.

3. Demark-Wahnefried W, Peterson BL, Winer EP, Marks L, Aziz N, Marcom PK, et al. Changes in weight, body composition, and factors influencing energy balance among premenopausal breast cancer patients receiving adjuvant chemotherapy. J Clin Oncol. 2001;19(9):2381-9.

4. Makari-Judson G, Judson $\mathrm{CH}$, Mertens WC. Longitudinal patterns of weight gain after breast cancer diagnosis: observations beyond the first year. Breast J. 2007;13(3):258-65.

5. Winkels RM, Beijer S, van Lieshout R, van Barneveld D, Hofstede $\mathrm{J}$, Kuiper J, et al. Changes in body weight during various types of chemotherapy in breast cancer patients. e-SPEN Journal. 2014; 9(1):e39-e44

6. Stenholm S, Harris TB, Rantanen T, Visser M, Kritchevsky SB, Ferrucci L. Sarcopenic obesity-definition, etiology and consequences. Curr Opin Clin Nutr Metab Care. 2008;11(6):693-700. doi: 10.1097 /MC0.0b013e328312c37d.

7. Baek SJ, Nam GE, Han KD, Choi SW, Jung SW, Bok AR, et al. Sarcopenia and sarcopenic obesity and their association with dyslipidemia in Korean elderly men: the 2008-2010 Korea National Health and Nutrition Examination Survey. J Endocrinol Invest. 2014; 37(3):247-60. doi: 10.1007/s40618-013-0011-3. Epub 2014 Jan 9.

8. Teixeira TF, Souza NC, Chiarello PG, Franceschini SC, Bressan J, Ferreira $\mathrm{CL}$, et al. Intestinal permeability parameters in obese patients are correlated with metabolic syndrome risk factors. Clin Nutr. 2012;31(5):735-40. doi: 10.1016/j.clnu.2012.02.009. Epub 2012 Mar 22

9. Zoico E, Rossi A, Di Francesco V, Sepe A, Olioso D, Pizzini F, et al. Adipose tissue infiltration in skeletal muscle of healthy elderly men: relationships with body composition, insulin resistance, and inflammation at the systemic and tissue level. J Gerontol A Biol Sci Med Sci. 2010;65(3):295-9. doi: 10.1093/gerona/glp155. Epub 2009 Oct 28.

10. O'Flanagan $\mathrm{CH}$, Bowers LW, Hursting SD. A weighty problem: metabolic perturbations and the obesity-cancer link. Horm Mol Biol Clin Investig. 2015;23(2):47-57. doi: 10.1515/hmbci-2015-0022.

11. McNeely ML, Campbell KL, Rowe BH, Klassen TP, Mackey JR, Courneya KS. Effects of exercise on breast cancer patients and survivors: a systematic review and meta-analysis. CMAJ. 2006; 175(1):34-41.

12. Blom WA, Lluch A, Stafleu A, Vinoy S, Holst JJ, Schaafsma G, et al. Effect of a high-protein breakfast on the postprandial ghrelin response. Am J Clin Nutr. 2006;83(2):211-20.

13. Batterham RL, Heffron H, Kapoor S, Chivers JE, Chandarana K, Herzog $\mathrm{H}$, et al. Critical role for peptide YY in protein-mediated satiation and body-weight regulation. Cell Metab. 2006;4(3):223-33.

14. Aragón F, Perdigón G, de LeBlanc AM. Modification in the diet can induce beneficial effects against breast cancer. World J Clin Oncol. 2014;5(3):455-64. doi: 10.5306/wjco.v5.i3.455.

15. Commane D, Hughes R, Shortt C, Rowland I. The potential mechanisms involved in the anti-carcinogenic action of probiotics. Mutat Res. 2005;591(1-2):276-89. Epub 2005 Aug 10.

16. Lin J, Manson JE, Lee IM, Cook NR, Buring JE, Zhang SM. Intakes of calcium and vitamin $\mathrm{D}$ and breast cancer risk in women. Arch Intern Med. 2007;167(10):1050-9.

17. Chen $\mathrm{P}$, Hu $\mathrm{P}$, Xie $\mathrm{D}$, Qin $\mathrm{Y}$, Wang $\mathrm{F}$, Wang $\mathrm{H}$. Meta-analysis of vitamin $\mathrm{D}$, calcium and the prevention of breast cancer. Breast Cancer Res Treat. 2010;121(2):469-77. doi: 10.1007/s10549-0090593-9. Epub 2009 0ct 23.

18. Knols R, Aaronson NK, Uebelhart D, Fransen J, Aufdemkampe G. Physical exercise in cancer patients during and after medical treatment: a systematic review of randomized and controlled clinical trials. J Clin Oncol. 2005;23(16):3830-42.

19. Schmitz KH, Ahmed RL, Hannan PJ, Yee D. Safety and efficacy of weight training in recent breast cancer survivors to alter body composition, insulin, and insulin-like growth factor axis proteins. Cancer Epidemiol Biomarkers Prev. 2005;14(7):1672-80. 
20. Demark-Wahnefried W, Rock CL. Nutrition-related issues for the breast cancer survivor. Semin Oncol. 2003;30(6):789-98.

21. de Rezende LF, Franco RL, de Rezende MF, Beletti PO, Morais SS, Gurgel MS. Two exercise schemes in postoperative breast cancer: comparison of effects on shoulder movement and lymphatic disturbance. Tumori. 2006;92(1):55-61.

22. Bamman MM, Clarke MS, Feeback DL, Talmadge RJ, Stevens BR, Lieberman SA, et al. Impact of resistance exercise during bed rest on skeletal muscle sarcopenia and myosin isoform distribution. J Appl Physiol (1985). 1998;84(1):157-63.

23. Schulte JN, Yarasheski KE. Effects of resistance training on the rate of muscle protein synthesis in frail elderly people. Int J Sport Nutr Exerc Metab. 2001;11 Suppl:S111-8.

24. Battaglini CL, Mills RC, Phillips BL, Lee JT, Story CE, Nascimento $M G$, et al. Twenty-five years of research on the effects of exercise training in breast cancer survivors: A systematic review of the literature. World J Clin Oncol. 2014;5(2):177-90. doi: 10.5306/ wjco.v5.i2.177.

25. Ciampolini M, Lovell-Smith D, Sifone M. Sustained self-regulation of energy intake. Loss of weight in overweight subjects. Maintenance of weight in normal-weight subjects. Nutr Metab (Lond). 2010;7:4. doi: 10.1186/1743-7075-7-4.

26. Campbell WW, Trappe TA, Wolfe RR, Evans WJ. The recommended dietary allowance for protein may not be adequate for older people to maintain skeletal muscle. J Gerontol A Biol Sci Med Sci. 2001; 56(6):M373-80.

27. Leidy HJ, Carnell NS, Mattes RD, Campbell WW. Higher protein intake preserves lean mass and satiety with weight loss in pre-obese and obese women. Obesity (Silver Spring). 2007;15(2):421-9.

28. Paddon-Jones D, Sheffield-Moore M, Urban RJ, Sanford AP, Aarsland A, Wolfe RR, et al. Essential amino acid and carbohydrate supplementation ameliorates muscle protein loss in humans during 28 days bedrest. J Clin Endocrinol Metab. 2004;89(9):4351-8.

29. Paddon-Jones D, Rasmussen BB. Dietary protein recommendations and the prevention of sarcopenia. Curr Opin Clin Nutr Metab Care. 2009;12(1):86-90. doi: 10.1097/MC0.0b013e32831cef8b

30. Demark-Wahnefried W, Kenyon AJ, Eberle P, Skye A, Kraus WE. Preventing sarcopenic obesity among breast cancer patients who receive adjuvant chemotherapy: results of a feasibility study. Clin Exerc Physiol. 2002;4(1):44-49.

31. Playdon M, Thomas G, Sanft T, Harrigan M, Ligibel J, Irwin M. Weight loss intervention for breast cancer survivors: a systematic review. Curr Breast Cancer Rep. 2013;5(3):222-246. Epub 2015 May 17.

32. Partridge AH, Burstein HJ, Winer EP. Side effects of chemotherapy and combined chemohormonal therapy in women with early-stage breast cancer. J Natl Cancer Inst Monogr. 2001;(30):135-42.

33. Ganz PA, Kwan L, Stanton AL, Bower JE, Belin TR. Physical and psychosocial recovery in the year after primary treatment of breast cancer. J Clin Oncol. 2011;29(9):1101-9. doi: 10.1200/JC0.2010. 28.8043. Epub 2011 Feb 7.

34. Heideman WH, Russell NS, Gundy C, Rookus MA, Voskuil DW. The frequency, magnitude and timing of post-diagnosis body weight gain in Dutch breast cancer survivors. Eur J Cancer. 2009;45(1): 119-26. doi: 10.1016/j.ejca.2008.09.003. Epub 2008 0ct 17.

35. Jakesz R, Jonat W, Gnant M, Mittlboeck M, Greil R, Tausch C, et al. Switching of postmenopausal women with endocrine-responsive early breast cancer to anastrozole after 2 years' adjuvant tamoxifen: combined results of ABCSG trial 8 and ARNO 95 trial. Lancet. 2005;366(9484):455-62.

36. Mialich MS, Sicchieri JMF, Junior AAJ. Analysis of body composition: A critical review of the use of bioelectrical impedance analysis. Int $J$ Clin Nutr. 2014;2(1):1-10. 